\title{
Vector-like contributions from Optimized Perturbation in the Abelian Nambu-Jona-Lasinio model for cold and dense quark matter
}

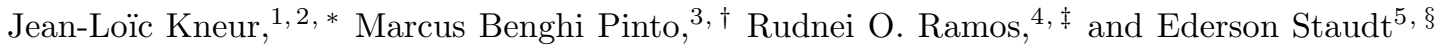 \\ ${ }^{1}$ CNRS, Laboratoire Charles Coulomb UMR 5221, F-34095, Montpellier, France \\ ${ }^{2}$ Université Montpellier 2, Laboratoire Charles Coulomb UMR 5221, F-34095, Montpellier, France \\ ${ }^{3}$ Departamento de Física, Universidade Federal de Santa Catarina, 88040-900 Florianópolis, Santa Catarina, Brazil \\ ${ }^{4}$ Departamento de Física Teórica, Universidade do Estado do Rio de Janeiro, 20550-013 Rio de Janeiro, RJ, Brazil \\ ${ }^{5}$ Departamento de Física, Universidade Federal do Amapá, 68902-280 Macapá, Amapá, Brazil
}

\begin{abstract}
Two-loop corrections for the standard Abelian Nambu-Jona-Lasinio model are obtained with the Optimized Perturbation Theory (OPT) method. These contributions improve the usual mean-field and Hartree-Fock results by generating a $1 / N_{c}$ suppressed term, which only contributes at finite chemical potential. We take the zero temperature limit observing that, within the OPT, chiral symmetry is restored at a higher chemical potential $\mu$, while the resulting equation of state is stiffer than the one obtained when mean-field is applied to the standard version of the model. In order to understand the physical nature of these finite $N_{c}$ contributions, we perform a numerical analysis to show that the OPT quantum corrections mimic effective repulsive vector-vector interaction contributions. We also derive a simple analytical approximation for the mass gap, accurate at the percent level, matching the mean-field approximation extended by an extra vector channel to OPT. For $\mu \gtrsim \mu_{c}$ the effective vector coupling matching OPT is numerically close (for the Abelian model) to the Fierz-induced Hartree-Fock value $G /\left(2 N_{c}\right)$, where $G$ is the scalar coupling, and then increases with $\mu$ in a well-determined manner.
\end{abstract}

In press Int. J. Mod. Phys. E (2012)

PACS numbers: 12.39.Fe,21.65.-f,11.15.Tk,11.15.Pg

\section{INTRODUCTION}

Effective models are extensively used to understand the physics of strong interactions. This is particularly true in the study of the structure of the phase diagram of Quantum Chromodynamics (QCD), specially in the low temperature (energy) and high density region, which is supposedly almost inaccessible through the present day lattice techniques that make direct use of QCD. In this respect, the use of effective models for quark interactions, like for example the Nambu-Jona-Lasinio (NJL) type of models [1] has proven to be extremely helpful to improve the understanding of the phase structure for strongly interacting matter.

With NJL type of models the phase structure of QCD has been explored mostly in terms of the well known $1 / N_{c}$ expansion, whose leading contribution represents the large- $N_{c}(\mathrm{LN})$ approximation and also corresponds to the meanfield-approximation (MFA) 2]. Recently, the alternative Optimized Perturbation Theory (OPT) method has been applied to the NJL model [3] and results beyond the MFA have been explicitly obtained for quantities related to the QCD phase structure. This application has been extended to the strong coupling and small current mass regime in order to investigate the critical line on the chemical potential-current mass plane [4].

For example, it has been observed that, at $T=0$, the OPT predicts that the first-order phase transition takes place at a chemical potential value that is higher than the one predicted by the MFA, thus, chiral symmetry breaking is enhanced. At this point it is interesting to remark that this trend is also observed when the MFA is applied to the NJL augmented by a repulsive vector-vector interaction term, $-G_{V}\left(\bar{\psi} \gamma^{\nu} \psi\right)^{2}$. It is then plausible to imagine that the OPT $1 / N_{c}$ correction captures some of the physics associated with this kind of interaction, which plays a major role at finite densities. The aim of the present work is to address this possibility by performing a numerical comparison between the OPT results, obtained from the standard NJL model, and the MFA results, obtained with the extended

\footnotetext{
*Electronic address: jlkneur@univ-montp2.fr

${ }^{\dagger}$ Electronic address: marcus@fsc.ufsc.br

¥Electronic address: rudnei@uerj.br

$\S$ Electronic address: tipller@yahoo.com
} 
version of model, such that the role of the quantum corrections generated by the former approximation can be better understood.

Although of academic interest only, the Abelian version of the NJL is well suited for such a task, since in this case, due to cancellation of terms between scalar and pseudo-scalar contributions at the exchange level [2], only a $\mu$ dependent contribution survives, allowing for a clearer comparison. Therefore, the temperature does not play a very important role in the present study and we, consequently, can restrict the analysis to the $T=0$ case. By adjusting the value of the vector-vector coupling in the MFA we will perform a numerical analysis exhibiting that the OPT results can be approximately well reproduced, near criticality and quite beyond, at the value $G_{V} \simeq G /\left(2 N_{c}\right)$, where $G$ is the scalar coupling. As we shall discuss, this could be anticipated by comparing the OPT and MFA relations for the free energy density. We then argue that one of the OPT main effects is to induce a new type of physics within the standard version of the NJL, by radiatively generating a vector-vector type of $1 / N_{c}$ correction that mainly affects the finite $\mu$ results. The possible generalization of these findings and their consequences for general four-fermion effective models, such as the non-Abelian NJL model as well as the Gross-Neveu model, and perhaps even for QCD, is currently being investigated. In this respect, the present investigation, that is carried out in this simple case, is important in its own right, since it offers the necessary framework to pursue such generalizations. We should also notice that the particular value $G_{V}=G /\left(2 N_{c}\right)$ at the level of our approximations just corresponds to the Fierz-induced Hartree-Fock (HF) approximation, which is not surprising since the OPT at first order involves corrections topologically similar to Fierz exchange terms. However, when considering the $\mu$-dependence, as we shall illustrate the OPT incorporates more corrections than simply Fierz-exchange terms, and the $G_{V}$ value best fitting near criticality is merely a numerical accident, only valid for the Abelian model. An additional bonus of our study is thus to produce a simple general analytical comparison of the MFA with vector-vector interaction approximation with the OPT one, from which we will be able to show the differences and advantages of the OPT at first-order over the HF approximation.

The remaining of this work is organized as follows. In Sec. II we derive the free energy density using the OPT formalism. In Sec. III we perform a numerical comparison between the OPT and the MFA results for the thermodynamical quantities at $T=0$. The analytical comparison of the OPT results with those coming from MFA with $G_{V} \neq 0$ is studied in Sec. IV] Our conclusions are presented in Sec. D.

\section{THE OPT FREE ENERGY FOR THE ABELIAN NJL MODEL}

The simplest version of the Abelian NJL model is described by a Lagrangian density for fermionic fields given by [1]

$$
\mathcal{L}=\bar{\psi}\left(i \not \partial-m_{c}\right) \psi+G\left[(\bar{\psi} \psi)^{2}+\left(\bar{\psi} i \gamma_{5} \psi\right)^{2}\right]
$$

where $\psi$ in general represents a $N_{c}$-plet quark of just one flavor with current mass $m_{c}$. Therefore, here, we consider the one-flavor model with (global) $U(1)$ symmetry. In the OPT interpolation prescription [7] applied in the case of four-fermion theories [3, 5, 6], one starts by deforming the original theory with the replacements $m_{c} \rightarrow m_{c}+(1-\delta) \eta$ and $G \rightarrow \delta G$ in Eq. (2.1) where $\delta$ is the alternative perturbative expansion parameter that determines the order at which the OPT expansion is carried out and $\eta$ is an arbitrary mass parameter to be set in a variational way through an appropriate optimization procedure at a given order in the OPT. The typical optimization procedure used in the many previous applications and that we also adopt here, is the Principle of Minimal Sensitivity (PMS), defined by [8]

$$
\left.\frac{d \mathcal{P}^{(k)}}{d \eta}\right|_{\bar{\eta}, \delta=1}=0
$$

where $\mathcal{P}^{(k)}$ is some physical quantity calculated up to order $k$ in the OPT. From the above prescriptions to build the interpolated model and rewriting the quartic interaction in (2.1) through a Hubbard-Stratonovich transformation introducing auxiliary fields, $\sigma$ and $\pi$, we obtain the interpolated Lagrangian density in the OPT formalism,

$$
\mathcal{L}=\bar{\psi}\left\{i \not \partial-\left(m_{c}+\eta\right)+\delta\left[(\eta-\sigma)-i \gamma_{5} \pi\right]\right\} \psi-\delta \frac{1}{4 G}\left(\sigma^{2}+\pi^{2}\right)
$$

Note from the interpolated theory Eq. (2.3) that the original Lagrangian is recovered for $\delta=1$, but at any finite order $k$ in the OPT, any perturbative result evaluated with Eq. (2.3) becomes dependent on $\eta$. Fixing it through the optimization procedure (2.2), new kinds of contributions beyond MFA are embodied in the non-trivial optimal $\eta$-dependence. 
For the studies involving the thermodynamics and phase structure of a given field theory model, the most appropriate quantity to be optimized has been shown [3, 5, 6] to be the effective potential (or free energy density), $\mathcal{F}$. Applying the optimization procedure (2.2) to $\mathcal{F}$ evaluated to some order $k$ in the OPT, we can then access the system's phase structure in a nonperturbative way beyond MFA. From the interpolated Lagrangian density, Eq. (2.3), we can immediately read the corresponding Feynman rules and then perform a perturbative expansion of the free energy density in powers of $\delta$. Then, the free energy density in the $\sigma_{c}$ direction (using that $\langle\sigma\rangle=\sigma_{c}$ and $\langle\pi\rangle=0$ ) reads $[3]$

$$
\begin{aligned}
\mathcal{F}^{\mathrm{OPT}} & =\frac{\sigma_{c}^{2}}{4 G}+2 i N_{c} \int \frac{d^{4} p}{(2 \pi)^{4}} \ln \left[-p^{2}+\left(m_{c}+\eta\right)^{2}\right]-4 i \delta N_{c} \int \frac{d^{4} p}{(2 \pi)^{4}} \frac{\left(m_{c}+\eta\right)\left(\eta-\sigma_{c}\right)}{-p^{2}+\left(m_{c}+\eta\right)^{2}} \\
& -8 \delta G N_{c}\left[\int \frac{d^{4} p}{(2 \pi)^{4}} \frac{p_{0}}{-p^{2}+\left(m_{c}+\eta\right)^{2}}\right]^{2} .
\end{aligned}
$$

All the integrals in Eq. (2.4) are to be interpreted in the Matsubara's finite temperature formalism with

$$
\int \frac{d^{4} p}{(2 \pi)^{4}} \equiv \frac{i}{\beta} \sum_{n=-\infty}^{+\infty} \int \frac{d^{3} p}{(2 \pi)^{3}}
$$

and quadri-momenta given as $p=\left(i \omega_{n}+\mu, \mathbf{p}\right)$, where $\omega_{n}=(2 n+1) \pi T, n=0, \pm 1, \pm 2, \ldots$, are the Matsubara frequencies for fermions. By performing the sum over these frequencies (see e.g. Ref. [3]), one obtains

$$
\mathcal{F}^{\mathrm{OPT}}=\frac{\sigma_{c}^{2}}{4 G}-2 N_{c} I_{1}(\mu, T, \eta)+2 \delta N_{c}\left(\eta+m_{c}\right)\left(\eta-\sigma_{c}\right) I_{2}(\mu, T, \eta)+2 \delta G N_{c} I_{3}^{2}(\mu, T, \eta),
$$

where we have defined, for convenience, the following basic integrals:

$$
\begin{gathered}
I_{1}(\mu, T, \eta)=\int \frac{d^{3} p}{(2 \pi)^{3}}\left\{E_{p}+T \ln \left[1+e^{-\left(E_{p}+\mu\right) / T}\right]+T \ln \left[1+e^{-\left(E_{p}-\mu\right) / T}\right]\right\} \\
I_{2}(\mu, T, \eta)=\int \frac{d^{3} p}{(2 \pi)^{3}} \frac{1}{E_{p}}\left[1-\frac{1}{e^{\left(E_{p}+\mu\right) / T}+1}-\frac{1}{e^{\left(E_{p}-\mu\right) / T}+1}\right]
\end{gathered}
$$

and

$$
I_{3}(\mu, T, \eta)=\int \frac{d^{3} p}{(2 \pi)^{3}}\left[\frac{1}{e^{\left(E_{p}-\mu\right) / T}+1}-\frac{1}{e^{\left(E_{p}+\mu\right) / T}+1}\right]
$$

where $E_{p}^{2}=\mathbf{p}^{2}+\left(\eta+m_{c}\right)^{2}$. Here, as in Ref. [3], only the divergent integrals occurring in Eqs. (2.7) and (2.8) are regularized by a sharp non-covariant three-dimensional momentum cutoff, $\Lambda$, so that the Stefan-Boltzmann limit is observed. Note that the second term in Eq. (2.4) corresponds to a gas of free fermions, whose mass has been dressed by $\eta$, while the third term represents tadpole type of contributions, proportional to the quark condensate, $\langle\bar{\psi} \psi\rangle$. The last term in Eq. (2.4), which is proportional to the square of the quark number density, $\left\langle\psi^{+} \psi\right\rangle^{2}$, comes from the two-loop terms in a next-to-leading order correction in the $1 / N_{c}$ expansion, which can be easily seen by redefining $G \rightarrow G / N_{c}$. This term is responsible for generating the vector type of corrections.

In order to perform our evaluations, we need to consider the general PMS equation (2.2), which can be conveniently expressed in the form [3]

$$
\left\{\left(\eta-\sigma_{c}\right)\left[1+\left(\eta+m_{c}\right) \frac{d}{d \eta}\right] I_{2}+2 G I_{3} \frac{d}{d \eta} I_{3}\right\}_{\eta=\bar{\eta}}=0 .
$$

One observes from Eqs. (2.6) and (2.9) that the first $1 / N_{c}$ correction $^{1}$ (represented by the last term) is only relevant when $\mu \neq 0$, as it vanishes for $\mu=0$. In the latter case, the PMS condition gives $\bar{\eta}=\sigma_{c}$, merely reproducing the well

\footnotetext{
${ }^{1}$ In the non-Abelian case there is also a $1 / N_{c}$ contribution proportional to $I_{2}^{2}$, which vanishes in the Abelian case [3] .
} 
known MFA results. Thus, the relevant contribution arises when $\mu \neq 0$. Since we are mainly interested in the phase structure of the model and on the effects of the OPT on it, one basic quantity of interest is thus the thermodynamical potential, $\Omega$, whose relation to the free energy is given by $\Omega=\mathcal{F}\left(\bar{\sigma}_{c}\right)$. The optimized order parameter, $\bar{\sigma}_{c}$, is determined from the gap equation generated by minimizing $\mathcal{F}$ with respect to the classical field, $\sigma_{c}$. From Eq. (2.6) we obtain that

$$
\bar{\sigma}_{c}=4 G N_{c}\left(\bar{\eta}+m_{c}\right) I_{2}(\mu, T, \bar{\eta})
$$

which can be inserted in the PMS equation to determine the optimized pressure.

\section{MATCHING THE OPT CORRECTIONS IN TERMS OF A VECTOR INTERACTION}

The effects due to the OPT first-order corrections for cold and dense quark matter can now be studied for the case of finite density effects $(\mu \neq 0)$ at zero temperature which is easily achieved by taking the limit $T \rightarrow 0$ in the thermal integrals [3]. Physically, this situation is relevant in studies related to neutron stars for example. This is also the regime where lattice techniques face more problems with the sign problem. Having obtained the optimized pressure one can easily obtain the quark density number, $\rho=d P / d \mu$, and by taking into account both the gap and PMS equations. The energy density is given as usual by $\varepsilon=-P+\mu \rho$ and allows us to obtain the equation of state (EoS).

Now, in order to carry out comparisons with the MFA let us recall that, as emphasized in Refs. 9 11], the introduction of a repulsive vector-vector interaction term of the form $-G_{V}\left(\bar{\psi} \gamma^{\nu} \psi\right)^{2}$ in Eq. (2.1) is also allowed by chiral symmetry. Such a term can become important at finite densities, generating a saturation mechanism, depending on the vector coupling strength, that provides better matter stability. Also, as far as phase transitions are concerned, it can influence the size of the first-order transition region and, hence, the location of the (tri)critical point. As already mentioned, the main goal of the present work is to compare the OPT results obtained at $G_{V}=0$, with the MFA results for $G_{V} \neq 0$ and whose free energy density is given by [9]

$$
\mathcal{F}^{\mathrm{MFA}}=\frac{\left(M_{\mathrm{MFA}}-m_{c}\right)^{2}}{4 G}-2 N_{c} I_{1}\left(\tilde{\mu}, T, M_{\mathrm{MFA}}\right)-4 G_{V} N_{c}^{2} I_{3}^{2}\left(\tilde{\mu}, T, M_{\mathrm{MFA}}\right),
$$

where $M_{\mathrm{MFA}}$ is the mass gap in MFA. In this case, one has to simultaneously solve the equations

$$
\tilde{\mu}=\mu-4 G_{V} N_{c} I_{3}\left(\tilde{\mu}, T, M_{\mathrm{MFA}}\right)
$$

and

$$
M_{\mathrm{MFA}}=m_{c}+4 G N_{c} M_{\mathrm{MFA}} I_{2}\left(\tilde{\mu}, T, M_{\mathrm{MFA}}\right)
$$

For our purpose, it is crucial to notice that the combined effects of $\tilde{\mu}$, defined by Eq. (3.2), and of the $-4 G_{V} N_{c}^{2} I_{3}^{2}$ term in Eq. (3.1), is to produce a net positive contribution approximately $\sim 4 G_{V} N_{c}^{2} I_{3}^{2}$ (for $\mu \gg M$ ) to the the free energy [12, 13]. This last term turns out to be of the same form as the OPT finite $N_{c} \operatorname{correction}$ term, $2 G N_{c} I_{3}^{2}$, so that one may guess that results obtained with this approximation should be close to those obtained with the MFA at $G_{V}$ values of the order $G /\left(2 N_{c}\right)$, but not the same (see detailed discussion in Sec. IV). This is precisely what we will check now in a numerical fashion by considering the EoS.

Following the standard procedure for the NJL model, we set the OPT parameter values to $\Lambda=620 \mathrm{MeV}, G \Lambda^{2}=4.44$ with $m_{c}=5.0 \mathrm{MeV}$, so that at $T=0$ one matches by convention the "physical" values of $f_{\pi}=92.4 \mathrm{MeV}, m_{\pi}=$ $135 \mathrm{MeV}$, while predicting $M=341 \mathrm{MeV}$, and $-\langle\bar{\psi} \psi\rangle^{1 / 3}=191 \mathrm{MeV}$ [14. One subtlety is that the $m_{c}$ value thus determined for OPT is strictly speaking slightly different from the corresponding MFA $m_{c}$, when obtained consistently from fitting the same pion mass value: this is due to the fact that the OPT gives $G^{2}$ correction to the relation between $m_{c}$ and $m_{\pi}$ [3]. More precisely, one finds $m_{c}^{M F A} \sim 5.36 \mathrm{MeV}$ instead of $m_{c}^{O P T} \sim 5.0 \mathrm{MeV}$ for the same $\Lambda=620 \mathrm{MeV}$ input. (In contrast the $G$ value being obtained from $f_{\pi}$ is not changed from MFA to OPT, since for the $U(1)$ model, $f_{\pi}$ receives no OPT corrections at $G^{2}$ order). Within the MFA, we will also use the following representative values for the vector coupling, $G_{V}=0,3 G /\left(8 N_{c}\right), G /\left(2 N_{c}\right)$ and $3 G /\left(4 N_{c}\right)$.

Figure 1] shows the quark effective mass as function of the chemical potential $\mu$, obtained with the OPT and the MFA for the four different $G_{V}$ values. First, one notes that the $\mu_{c}$ value at which chiral symmetry is restored, via 


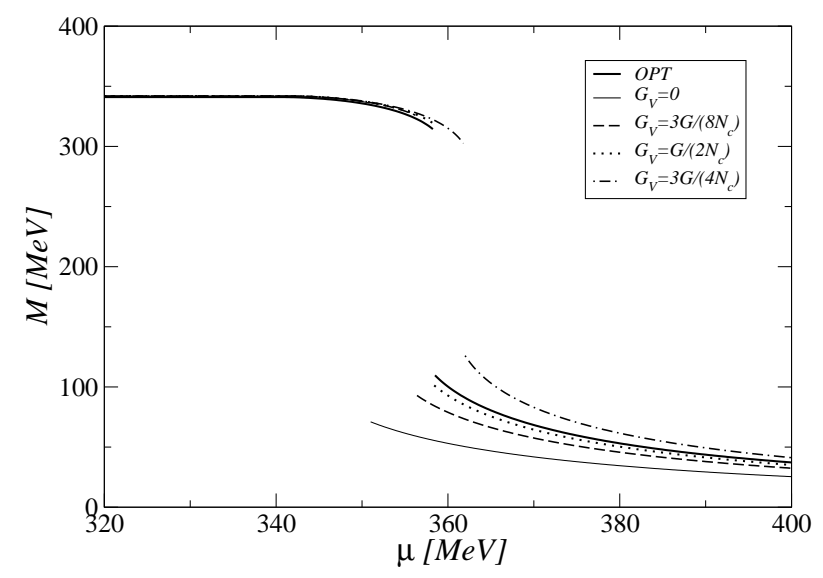

FIG. 1: Effective quark mass (at $T=0$ ) as a function of the chemical potential.

TABLE I: The critical chemical potential $\mu_{c}(T=0)$ in the OPT and in the MFA (for the different values of $G_{V}$ ).

\begin{tabular}{c|c|c|c|c|c}
\hline$G_{V}$ & 0 & $3 G /\left(8 N_{c}\right)$ & $G /\left(2 N_{c}\right)$ & $3 G /\left(4 N_{c}\right)$ & $\mathrm{OPT}\left(G_{V}=0\right)$ \\
\hline$\mu_{c}(\mathrm{MeV})$ & 350.9 & 356.3 & 358.3 & 361.9 & 358.5 \\
\hline
\end{tabular}

a first order transition, increases with $G_{V}$ in the MFA. The critical chemical potential value predicted by the MFA with $G_{V}=G /\left(2 N_{c}\right)$ agrees the better with the OPT prediction, as we can see also from the results shown in Tab. [.

The pressure subtracted by the $\mu=0$ value, $P(\mu)-P(0)$, as a function of the chemical potential is shown in Fig. 22, We also see from the results that the OPT prediction is very close to the MFA with $G_{V}=G /\left(2 N_{c}\right)$ near criticality, but the OPT curve is less steep than the MFA one and tends to deviate towards higher values of $G_{V}$ as $\mu$ increases. Thus, one may expect that the baryon density predicted by the OPT to be smaller at high $\mu$. This expectation is confirmed by the results shown in Fig. 3, where we show the baryon density as a function of the chemical potential. The same behavior is also reflected in Fig. [4 where is shown the energy density as a function of $\mu$. Again, one sees that the OPT and the MFA with $G_{V}=G /\left(2 N_{c}\right)$ are in best agreement around criticality, as shown by the results in Table【. while at higher values of $\mu$ the OPT result interpolates between this and higher effective $G_{V}$ values. This behavior of the OPT results at larger chemical potential can be predicted if we compare the OPT expression for the free energy density, Eq. (2.6), with the one in the MFA approximation, Eq. (3.1). Besides of the nontrivial dependence of the expression, in the OPT case, with the variational parameter $\eta$, there is also the additional contribution with the $I_{2}$ term in Eq. (2.6). All these contributions from the OPT tend to favor a comparatively larger value of $G_{V}$ in the MFA case, for higher values of the chemical potential, when trying to match the OPT results with the ones within the MFA with $G_{V} \neq 0$. In the next section we will trace this behaviour by deriving a more analytical connection between the OPT and such an effective mean field vector-vector coupling. Finally, this behavior of the OPT compared with the MFA case with a non vanishing value of $G_{V}$, also reflects on the EoS. The EoS results for the different cases are shown in Fig. 5 and serve to illustrate how the OPT corrections produces a stiffer equation of state for this version of the model [3].

\section{A SIMPLE ANALYTICAL APPROXIMATION}

We shall try to get a deeper insight about our previous numerical results by examining a simple well-motivated approximation. As we will see, it will essentially explain the bulk of the OPT results versus the MFA ones with extra vector coupling, explaining in particular the rather accidental best OPT matching by the $\mathrm{HF} G_{V}=G /\left(2 N_{c}\right)$ value near criticality. First, as it is well-known, the large- $N_{c}$ (or MFA) is also equivalent to the traditional Hartree approximation, which only incorporates effects from direct (tadpole) contributions, while exchange terms are also considered within HF. Indeed, the value $G_{V}=G /\left(2 N_{c}\right)$ is just the vector coupling one obtains by a Fierz transformation of the original interaction, Eq. (2.1). Therefore, Eq. (3.1) with this value of $G_{V}$ just corresponds to the HF approximation. Similarly, the two-loop induced first order OPT corrections to the free energy incorporate the structure given by $1 / N_{c}$ exchangegraph of the Fierz type. Indeed for $\mu=0$ (and $T=0$ ) the OPT results are generically consistent [3] with HF ones 


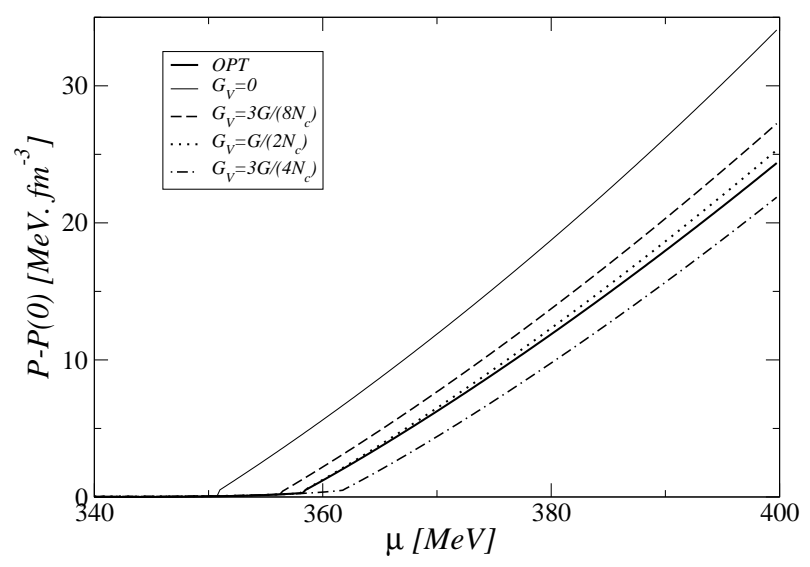

FIG. 2: The pressure (subtracted by the value at $\mu=0$ ) as a function of the chemical potential.

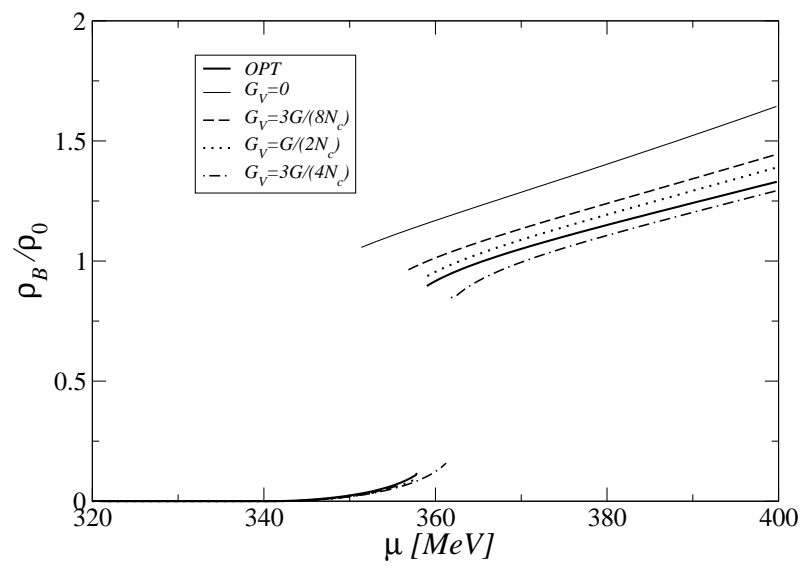

FIG. 3: Baryon number density, $\rho_{B}=\rho / 3$, in units of nuclear matter density $\left(\rho_{0}=0.17 \mathrm{fm}^{-3}\right)$, as a function of the chemical potential.

(up to higher $1 / N_{c}^{2}$ corrections originating from the optimization of the mass $\eta$ ). Note however that in the Abelian case, $M_{\mathrm{HF}}$ is identical to $M_{\mathrm{MFA}}$, due to the exact cancellation of the $1 / N_{c}$ scalar and pseudo-scalar contributions. However, as far as the $\mu$-dependence is concerned, OPT corrections also induce extra terms, as is already clear from Eq. (2.10). Comparing the set of equations above one sees that nonperturbative information concerning a vector type of interaction is being taken into account by both approximations in a different way. This becomes clear if one recalls that the $I_{3}$ integral roughly represents the quark number density, $\left\langle\psi^{+} \psi\right\rangle$, while the integral $I_{2}$ roughly represents the scalar condensate, $\langle\bar{\psi} \psi\rangle$. The last term of Eq. (2.10) explicitly displays that $I_{3}$ is also considered in a nonperturbative fashion.

In order to understand further beyond purely numerical results the origin of these differences, let us have a deeper look at the expressions for the MFA and OPT mass gap expressions, respectively given by Eq. (3.3) with (3.2) for the former (where $I_{2}$ and $I_{3}$ are to be evaluated at $\tilde{\mu}$ ), and Eqs. (2.11) and (2.10) for the latter. For $\mu>\mu_{c}$ a simple but crucial observation is that the behavior of both expressions is essentially driven by the explicit chiral symmetry breaking mass $m_{c}$. Indeed, for $m_{c}=0$, one would have $M_{M F A}\left(G_{V}\right)\left(\mu>\tilde{\mu}_{c}\right) \equiv 0$ and $M_{O P T}\left(\mu>\mu_{c}\right) \equiv 0$ consistently. Now, since $m_{c}$ is small as compared to all other scales, we may expect that to a good approximation the mass gap could be well approximated by a first-order expansion in $M$, next to be solved by a self-consistent equation (becoming in that case a trivial first-order equation):

$$
M \simeq m_{c}+\beta(\mu, \Lambda, G, \cdots) M+\mathcal{O}\left(M^{3}\right)
$$

where $\beta(\mu, \Lambda, G, \cdots)$ is the coefficient obtained from this first-order expansion. We also note that the next-order term 


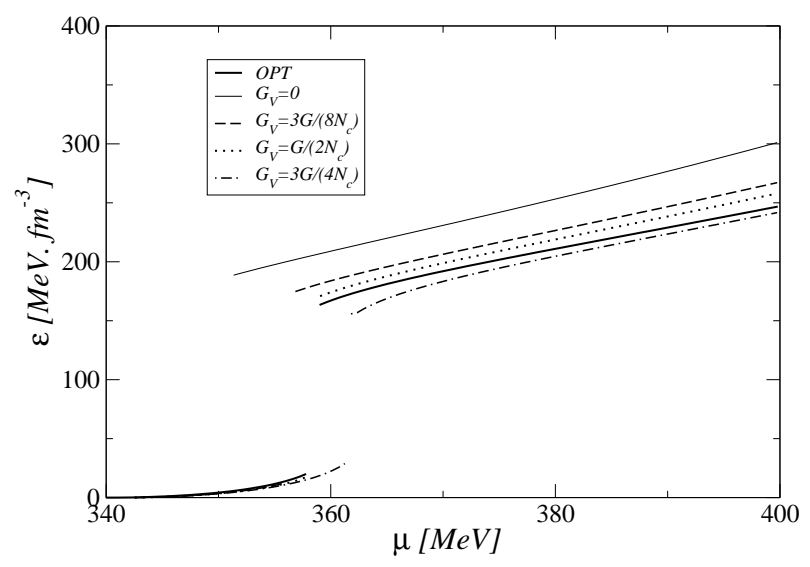

FIG. 4: Energy density as a function of the chemical potential.

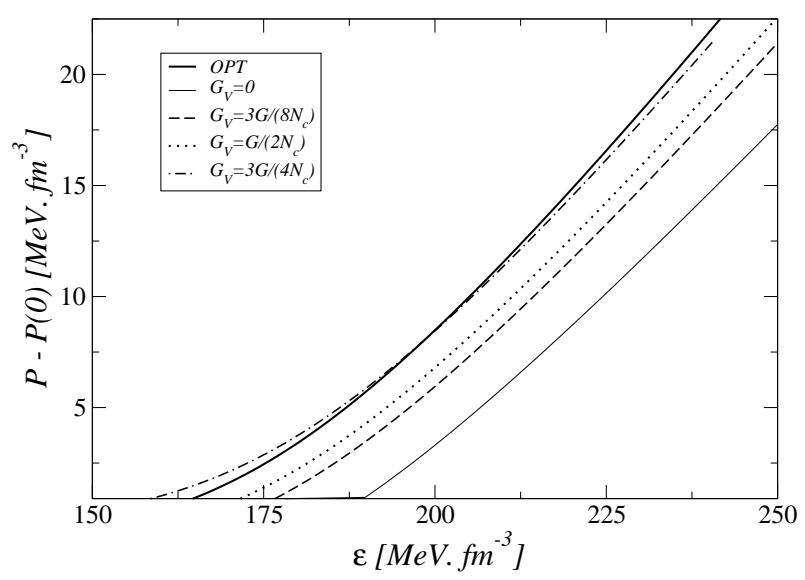

FIG. 5: Equation of state for quark matter (pressure as a function of energy density).

in the expansion in Eq. (4.1) is of order $M^{3}$.

Explicitly, we obtain after some simple algebra:

$$
M_{M F A}\left(G_{V}\right) \simeq m_{c}+\frac{N_{c} G}{\pi^{2}}\left(\Lambda^{2}-\tilde{\mu}^{2}\right) M_{M F A}+\mathcal{O}\left(M^{3}\right)
$$

where from Eq. (3.2),

$$
\tilde{\mu}=\mu-2 \frac{G_{V} N_{c}}{3 \pi^{2}} \tilde{\mu}^{3}+\mathcal{O}\left(\tilde{\mu} M^{2}\right),
$$

and

$$
M_{O P T} \simeq m_{c}+\frac{N_{c} G}{\pi^{2}}\left[\left(\Lambda^{2}-\mu^{2}\right)+\frac{2}{3 N_{c}} \frac{\mu^{4}}{\left(\Lambda^{2}-\mu^{2}\right)}\right] M_{O P T}+\mathcal{O}\left(M^{3}\right),
$$

respectively. The gap equation solutions are thus trivially given as

$$
M_{O P T}=m_{c}\left(1-\beta_{O P T}(\mu, \Lambda, G, \cdots)\right)^{-1},
$$


and similarly for $M_{M F A}\left(G_{V}\right)$. Note that those approximations both turn out to be accurate at the percent level, as compared to the exact numerical solutions, for a large range of $\mu_{c} \lesssim \mu \ll \Lambda$ values. This is largely due to the very suppression by higher $1 /\left(\Lambda^{2}-\mu^{2}\right)$ powers of higher order coefficients of these expansions in powers of $M$.

Now defining generically $G_{V}=\alpha G / N_{c}, \alpha$ will depend essentially on $\mu$ and this is what we would like to determine next. Before that, we observe that, at least for rather moderate values of $G_{V} \sim \mathcal{O}(G)$, Eq. (3.2) when solved exactly actually gives a moderate shift of $\tilde{\mu}$ with respect to $\mu$, giving $\tilde{\mu}$ slightly smaller than $\mu$ by only a few percent. So $\mu_{c}$ is well approximated by restricting Eq. (4.3) at first iteration, i.e., first $G_{V}$ order. This allows to derive a simple relation to determine the relevant coefficient $\alpha$ above for the $G_{V} / G$ ratio, as a function of $\mu$ and the other parameters. From the matching $M_{M F A}=M_{O P T}$, then from Eqs. (4.2) and (4.4) and using Eq. (4.3) at first iteration, but solving exactly the (quadratic) equation for $\alpha$, after a simple algebra one finds

$$
\begin{aligned}
\alpha & =\frac{3 \pi^{2}}{2 G \mu^{2}}\left[1-\sqrt{1-\frac{2 \mu^{2}}{3 N_{c}\left(\Lambda^{2}-\mu^{2}\right)}}\right] \\
& =\frac{\pi^{2}}{N_{c} G \Lambda^{2}}\left[1-\frac{\mu^{2}}{\Lambda^{2}}+\sqrt{\left(1-\frac{\mu^{2}}{\Lambda^{2}}\right)^{2}-\frac{2}{3 N_{c}} \frac{\mu^{2}}{\Lambda^{2}}\left(1-\frac{\mu^{2}}{\Lambda^{2}}\right)}\right]^{-1},
\end{aligned}
$$

which gives an explicit relation for the appropriate $G_{V}$ values needed to match the OPT results for arbitrary $\mu$ (only valid of course for $\mu \geq \mu_{c}$, and also for $\mu \ll \Lambda$ in principle, since reaching too close to the natural cutoff does not make much sense physically within the NJL effective model).

In deriving Eq. (4.6) it is assumed that both the OPT and MFA masses involve the very same current mass $m_{c}$, which is thus valid if $m_{c}$ is a common input to both approximations. However, as discussed above in Section III, the OPT $m_{c}$ value as determined consistently from fitting $m_{\pi}$ is slightly smaller than the corresponding MFA value. Although a rather modest change in $m_{c}$ it affects the results, due to the high sensitivity to $m_{c}$ for $\mu>\mu_{c}$, making the OPT mass gap lower by the same amount according to Eq. (4.5) above, as compared with the MFA mass gap for a given $G_{V}$ value. This partly delays the increase in the effective $G_{V}$ for increasing $\mu$ from Eq. (4.6), which can be easily taken into account and combined with the latter to extract a precise relation for $G_{V} / G$ appropriately modifying Eq. (4.6). Since $m_{c}^{O P T} / m_{c}^{M F A} \sim 0.93$, this gives a moderate modification to the relation (4.6) in practice, making the corresponding effective $G_{V}$ slightly smaller by about $10 \%$ for a given $\mu$. This fits remarkably well our numerical results for the mass gap obtained with the exact expressions, with only a few percent errors. For $\mu \sim \mu_{c} \simeq 360 \mathrm{MeV}$, i.e. very near criticality, one finds $G_{V} N_{c} / G \simeq 0.53$, which explains a posteriori why the results remain there very close to the $\mathrm{HF}$ value $G_{V} N_{c} / G \equiv 1 / 2$. However, it is also clear from the previous derivation that this is to some extent a numerical accident, resulting from a rather fortuitous combination of the Abelian model factors, $G \Lambda^{2} \simeq 4.44$ and $\mu_{c} / \Lambda$ values such that the term in parenthesis in Eq. (4.6) is $\sim 0.78$, plus the small correction from $m_{c}^{O P T} / m_{c}^{M F A}$ as above explained. Indeed for higher $\mu G_{V} / G$ grows first moderately and then more rapidly when $\mu$ increases towards $\Lambda$. For example, we obtain $G_{V}(\mu \sim 400 \mathrm{MeV}) \sim 0.6 G / N_{c}$ and $G_{V}(\mu \sim 500 \mathrm{MeV}) \sim 1.13 G / N_{c}$. This also illustrates the differences between OPT and HF or any other fixed $G_{V}$ value, since OPT mimics an effective $G_{V}(\mu)$ value. Finally, the behaviour of the other physical quantities in Figs. 2-5 may be understood semi-analytically along the same line of reasoning, though we skip the details here. The pressure in Fig. 2 follows roughly the same trend as the mass gap. As for the other quantities in Figs. 3-5, the more pronouced increase of the effective OPT-matching $G_{V}$ value at increasing $\mu$ values can be understood essentially by the enhanced $\mu$-dependence from the definition of the density.

\section{CONCLUSIONS}

In order to investigate the physical origin of the main effects produced by the OPT nonperturbative approximation, we have evaluated the free energy density for the Abelian NJL model. This version of the model is certainly less realistic than the non-Abelian ones, but being much simpler, it suits our purpose by providing an easier to analyze free energy. As usual, in the large- $N_{c}$ limit, the OPT reproduces the MFA result exactly, while at the next-to-leading $1 / N_{c}$ order as induced by OPT, the contributions from the scalar and pseudo-scalar channels cancel each other whenever $\mu=0$ (for this version of the model [3]). Nevertheless, the finite $N_{c}$ contributions may still be important for situations such as $T=0, \mu \neq 0$ (dense cold quark matter) and $T \neq 0, \mu \neq 0$ (hot and dense quark matter).

Considering the MFA predictions for the same version of the model one observes that the OPT prediction for the critical chemical potential value $\left(\mu_{c}\right)$, at $T=0$, is higher. However, as it is well known, the MFA applied to the NJL in the presence of an extra repulsive vector-vector interaction (proportional to the coupling $G_{V}$ ) also predicts higher $\mu_{c}$ values, as compared to the $G_{V}=0$ case. Then, comparing the mathematical structure of the free energy 
density provided by the OPT, with $G_{V}=0$, and the MFA, with $G_{V} \neq 0$, we show that the former approach radiatively generates an effective vector-like type of contribution which is $1 / N_{c}$ suppressed and, hence, does not appear in a large$N_{c}$ type of calculation. This comparison hinted to the value $G_{V}=G /\left(2 N_{c}\right)$ as the one for which both approximations could furnish similar results in a reasonable range of $\mu$ values near criticality. This has been successfully verified in both numerical and analytical manner, showing that the OPT predictions, obtained with one less parameter $\left(G_{V}\right)$, point out in the same direction as the ones produced by the MFA applied to the NJL with $G_{V} \neq 0$ and, thus, with a larger parameter space. In particular, the agreement for the value of $G_{V}=G /\left(2 N_{c}\right)$ is best near criticality, but we have shown by a simple analytical approximation how OPT incorporates corrections for higher $\mu$ matching higher $G_{V}$ values. Indeed it should be remarked that the very proximity, near criticality, of the OPT results with the simpler HF approximation, is not much a generic feature but a rather fortuitous accident of the very simple Abelian model here considered. In the $S U(2)$ version of the NJL model, the OPT exhibits [3] more departure from the HF approximation, and in other models also it generically captures corrections beyond HF (for instance in Ref. [15] results obtained from OPT at first order for the Gross-Neveu mass gap are very close to the full next-to-leading $1 / N$ corrections). Indeed an interesting follow up beyond the scope of the present work could be to examine similarly the correspondence between a generalized OPT-NJL SU(N) and extra four-fermion interactions in MFA.

The present application suggests that the OPT is able to bring new physics features, without introducing new parameters. This conclusion certainly deserves further investigation and we are carrying out a similar analysis for the case of general four-fermion theories in an attempt to estimate the consequences beyond the MFA for the QCD phase diagram with a special concern about the location of the critical point. These results will be presented elsewhere.

\section{Acknowledgments}

MBP would like to thank Kenji Fukushima for discussions. This work has been partially supported (MBP, ROR and ES) by Conselho Nacional de Desenvolvimento Científico e Tecnológico (CNPq) and by Coordenação de Aperfeiçoamento de Pessoal de Nível Superior (CAPES).

[1] Y. Nambu and G. Jona-Lasinio, Phys. Rev. 122 (1961) 345; 124 (1961) 246.

[2] S. P. Klevansky, Rev. Mod. Phys. 64 (1992) 649.

[3] J.-L. Kneur, M. B. Pinto and R. O. Ramos, Phys. Rev. C 81 (2010) 065205.

[4] L. Ferroni, V. Koch and M.B. Pinto, Phys. Rev. C 82 (2010) 055205.

[5] J.-L. Kneur, M. B. Pinto and R. O. Ramos, Phys. Rev. D 74 (2006) 125020.

[6] J.-L. Kneur, M. B. Pinto, R. O. Ramos and E. Staudt, Phys. Rev. D 76 (2007) 045020; Phys. Lett. B567 (2007) 136.

[7] A. Okopinska, Phys. Rev. D 35 (1987) 1835; M. Moshe and A. Duncan, Phys. Lett. B215 (1988) 352.

[8] P. M. Stevenson, Phys. Rev. D 23 (1981) 2961; Nucl. Phys. B 203 (1982) 472.

[9] M. Buballa, Phys. Rep. 407 (2005) 205.

[10] M. Buballa, Nucl. Phys. A 611 (1996) 393.

[11] V. Koch, T. S. Biro, J. Kunz, and U. Mosel, Phys. Lett. B 185 (1987) 1.

[12] K. Fukushima, Phys. Rev. D 77 (2008) 114028.

[13] K. Fukushima, Phys. Rev. D 78 (2008) 114019.

[14] E. Staudt, Ph.D Thesis, UFSC, 2009 (www.pgfsc.ufsc.br).

[15] J.-L. Kneur and A. Neveu, Phys. Rev. D 81 (2010) 125012. 